\title{
EFECTO DE LOS EMULSIFICANTES SOBRE LAS CARACTERÍSTICAS FíSICAS Y TEXTURALES DEL BUÑUELO
}

\section{EFFECT OF EMULSIFIERS ON PHYSICAL AND TEXTURAL CHARACTERISTICS OF BUÑUELO}

\begin{abstract}
Margarita M. Morales ${ }^{1}$, Eduardo Rodríguez-Sandoval ${ }^{2}$, Misael Cortes ${ }^{3}$
${ }^{1}$ Ing. Agroindustrial, C. Magister en Ciencia y Tecnología de Alimentos. Departamento de Ingeniería Agrícola y Alimentos. Facultad de Ciencias Agropecuarias. Universidad Nacional de Colombia. Núcleo el Volador. Medellín, Antioquia - Colombia, mmmoralesmo@unal.edu.co; ${ }^{2}$ Ing. Químico, Doctor en Ingeniería. Docente Asociado. Departamento de Ingeniería Agrícola y Alimentos. Facultad de Ciencias Agropecuarias. Universidad Nacional de Colombia. Núcleo el Volador. Medellín, Antioquia, edrodriguezs@unal.edu.co; ${ }^{3}$ Ing. Químico, Doctor en Ciencia y Tecnología de Alimentos. Docente Asociado. Departamento de Ingeniería Agrícola y Alimentos. Facultad de Ciencias Agropecuarias. Universidad Nacional de Colombia. Núcleo el Volador. Medellín. Antioquia, mcortesro@unal.edu.co
\end{abstract}

Rev. U.D.C.A Act. \& Div. Cient. 16(2): 417-425, Julio-Diciembre, 2013

\section{RESUMEN}

Los emulsificantes se utilizan como agentes antienvejecimiento en productos de panadería, proporcionando mayor vida útil. El objetivo de este estudio fue evaluar los efectos de dos emulsificantes, mono y diglicéridos, de los ácidos grasos esterificados, con ácido diacetil tartárico (DATEM) y estearoil lactilato de sodio (SSL), sobre las características reológicas de la masa de buñuelo y las propiedades físicas y químicas del producto, almacenado a temperatura ambiente. Se adicionó DATEM y SSL, en porcentajes de 0,5 y $1 \%$, con respecto al queso de la formulación. Las masas presentaron un efecto significativo sobre las constantes reológicas del modelo de Peleg, $\mathrm{k}_{1}$ y $\mathrm{k}_{2}$, obteniendo los menores valores para las muestras con emulsificante (DATEM o SSL), al 1\%. No se observó influencia significativa de los emulsificantes sobre las propiedades químicas y texturales (punción y dureza) del buñuelo. La cohesividad y la elasticidad del producto disminuyeron significativamente, al aumentar la concentración de SSL. El contenido de humedad, la actividad de agua y la fuerza máxima de punción disminuyeron durante el tiempo de almacenamiento. Por el contrario, la dureza de la miga aumentó en el periodo de almacenamiento.

Palabras clave: Producto libre de gluten, surfactante, SSL, DATEM, reología.

\section{SUMMARY}

Emulsifiers are used as anti-staling agents in bakery products, increased shelf life. The objective of this study was to evaluate the effects of two emulsifiers, diacetyl tartaric acid ester of mono- and diglycerides (DATEM) and sodium stearoyl lactylate (SSL), on the rheological characteristics of the buñuelo dough and on the physical and chemical properties of the product stored at room temperature. DATEM and SSL were added in percentages of $0,5 \%$ and $1 \%$ base on the cheese of the formulation. The dough samples showed a significant effect on the rheological model constants of Peleg, k1 and k2, and lower values were obtained for the samples with emulsifier (DATEM or SSL) at $1 \%$. The chemical and textural (puncture and hardness) properties of buñuelo were not influenced significantly by the emulsifiers. Cohesiveness and elasticity of the product decreased significantly as the concentration of SSL increased. Moisture content, water activity and puncture maximum force decreased during the storage time. On the other hand, the crumb hardness increased during the storage period.

Key words: Gluten free product, surfactant, SSL, DATEM, rheology.

\section{INTRODUCCIÓN}

El buñuelo es un producto tradicional de panadería obtenido, básicamente, de la mezcla de queso, de almidón agrio o fermentado de yuca, de fécula de maíz, agua o leche, pu- 
diendo también contener otros ingredientes, como huevos, azúcar y sal. Con ellos, se desarrolla una masa y se forman esferas, que se fríen en aceite, a una temperatura de 150 a $170^{\circ} \mathrm{C}$. El producto final tiene una corteza crujiente de color café, una miga suave, esponjosa y un olor y sabor característico. Además de sus propiedades organolépticas deseables es un producto libre de gluten, que lo coloca como alimento alternativo, para personas con enfermedad celiaca (Milde et al. 2009). Es de alto consumo en Colombia, si se tiene en cuenta que la venta de premezclas para buñuelo está por encima de 2250t/año, en donde participan empresas, como C.I. Productos el Nevado, Industrias del Maíz, Levapan, Fleishman, entre otros (Aristizábal \& Sánchez, 2007). De este producto nacional pocos estudios científicos se han realizado y su tecnología de fabricación no está estandarizada, siendo necesario el establecimiento de parámetros de calidad, para que la cadena de producción se pueda desarrollar.

El buñuelo, generalmente, se consume fresco, pero el fenómeno de envejecimiento se inicia muy rápidamente después de la fritura, presentando endurecimiento y rigidez, sobre todo si se mantienen a temperatura ambiente, durante más de 24 h. Hoy en día, la producción a gran escala y el aumento de consumidores que demandan una alta calidad y larga vida útil, han creado la necesidad del uso de aditivos alimentarios funcionales, tales como emulsificantes, enzimas, hidrocoloides y gomas, para lograr esos objetivos deseados (Koocheki et al. 2009).

Entre los aditivos alimentarios, los emulsificantes son ampliamente utilizados en la industria de panificación, para minimizar el envejecimiento del pan, mejorar la manipulación y fuerza de la masa, aumentar la tolerancia al tiempo de reposo y fermentación, entre otros aspectos (Chin et al. 2007). Ellos exhiben propiedades lipolíticas e hidrolíticas, reduciendo la tensión interfacial entre fases que, normalmente, no se mezclan. Se clasifican en dos clases: los que forman complejos con el almidón, favoreciendo la suavidad de la miga y previniendo el envejecimiento, como por ejemplo, los monoglicéridos y los que actúan en interacción con la proteína, que actúan como reforzadores de masa y aumentan la capacidad del gluten, para formar una película que retiene la producción de gas, tales como SSL (estearoil lactilato de sodio) y CSL (estearoil lactilato de calcio) (Chin et al. 2007).

El DATEM, SSL y CSL son los más utilizados en la industria de la panificación y facilitan la interacción de los lípidos con las proteínas y el almidón. Las ventajas más reconocidas de su uso son el incremento del volumen de pan y el mejoramiento de la textura de la miga. También se les asigna la formación de complejos insolubles con la amilosa, retardando así la capacidad de envejecimiento del pan (Beltrán-Orozco et al. 2007).
Existen pocas publicaciones sobre el efecto de los emulsificantes en las propiedades reológicas de la masa y la calidad de productos fritos, a base de almidón agrio de yuca y no existe información sobre el efecto de estos aditivos, en las características de la masa y la calidad del buñuelo. Solamente existen estudios que muestran el uso potencial de los emulsificantes en la industria de la panificación (Stampfli \& Nersten, 1995; Onyango et al. 2009; Sciarini et al. 2012; Gomes-Ruffi et al. 2012). Por lo tanto, el presente trabajo, se llevó a cabo con el fin de evaluar los efectos de dos emulsificantes, DATEM y SSL, sobre las características reológicas de la masa de buñuelo y las propiedades físicas y químicas del producto, almacenado a temperatura ambiente.

\section{MATERIALES Y MÉTODOS}

El ingrediente mayoritario es el queso tipo costeño, el cual, se elaboró en el Laboratorio de Productos Lácteos (Universidad Nacional de Colombia Sede Medellín). Los demás componentes utilizados en la preparación de buñuelo fueron comprados en el mercado local, tal como azúcar, polvo de hornear, huevo y leche. La fécula de maíz y el almidón agrio de yuca fueron adquiridos en Distribuidora JJJ (Medellín, Colombia). Los emulsificantes, DATEM (Nutrimuls ${ }^{\circledR}$ Datem) y SSL (Nutrimuls SSL 1078) fueron suministrados por Nutring S.A. (Buenos Aires, Argentina).

Para caracterizar el almidón agrio de yuca, se determinó la curva de empastamiento, utilizando un viscoamilógrafo RVA serie No. 4 (Newport Scientific, Warriewood, Australia) y se expresaron los valores de viscosidad en unidades del viscoamilógrafo rápido (RVU). Se preparó una suspensión de almidón en agua destilada, con una concentración del 5\% (p/p), exponiéndola a calentamiento y enfriamiento. La suspensión, se calentó hasta $90^{\circ} \mathrm{C}$ y se sostuvo a esa temperatura, por un período de $5 \mathrm{~min}$; posteriormente, se enfrió hasta $50^{\circ} \mathrm{C}$ y se mantuvo a esa temperatura, por $5 \mathrm{~min}$. La velocidad de calentamiento y de enfriamiento fue de $1,6^{\circ} \mathrm{C} / \mathrm{min}$. Se realizaron tres repeticiones por muestra. Cuatro parámetros característicos fueron medidos a partir de la curva de viscosidad vs. tiempo/temperatura, que se generó (Rodríguez-Sandoval et al. 2006): 1) Temperatura de inicio de la gelatinización (T gel): es la temperatura en la que se inicia el aumento de la viscosidad en la suspensión; 2) Viscosidad máxima (V max): es la máxima viscosidad alcanzada por la suspensión después, de la cual, la viscosidad empieza a descender; 3) Inestabilidad del gel (Breakdown): es la diferencia de viscosidad entre la viscosidad máxima y la viscosidad después de 5 min, a $90^{\circ} \mathrm{C}$; 4) Asentamiento (Setback): es la diferencia entre la viscosidad a $50^{\circ} \mathrm{C}$ y la viscosidad después de 5 min. a $90^{\circ} \mathrm{C}$.

El buñuelo, se elaboró con una formulación control, que tuvo como base la cantidad de queso y se distribuyó como 
sigue: queso tipo costeño, $100 \%$; fécula de maíz, $50 \%$; almidón fermentado de yuca, $15 \%$; azúcar, $6 \%$; polvo de hornear, $1 \%$; huevo, $10 \%$ y leche, $30 \%$. Los ingredientes queso, fécula de maíz, almidón agrio de yuca, azúcar y polvo de hornear, se mezclaron en una batidora (Profesional Series 600, KP26M1XER, KitchenAid, USA). Luego, se adicionó el huevo, medio plastificante para realizar una buena mezcla de los ingredientes; después, se añadió lentamente leche y se dejó mezclando por 5 min, hasta obtener una masa suave y homogénea. Por último, la masa se moldeó en forma de esferas de $30 \mathrm{~g}$ y se introdujo en un freidor eléctrico (Modelo EF-101, Wellborn, China), con aceite de maíz, a una temperatura de $160^{\circ} \mathrm{C} \pm 5^{\circ} \mathrm{C}$, durante $12 \mathrm{~min}$. El producto se extrajo del aceite y se secó en papel absorbente.

Después del freído, las muestras se dejaron reposar durante 1h, a temperatura ambiente y se determinaron: peso, diámetro, volumen, contenido de grasa y color. El procedimiento para realizar las muestras con emulsificantes (DATEM y SSL) fue el mismo, mezclando estos aditivos con los ingredientes secos, luego con el queso y, por último, se le adicionó el huevo y la leche. El porcentaje adicionado para cada emulsificante fue de 0,5 y $1 \%$, con respecto al queso de la formulación. Además, se evaluó su comportamiento en el almacenamiento, midiendo actividad de agua, contenido de humedad, dureza, fuerza de punción, elasticidad y cohesividad a 24, 48 y 72h. Siete muestras se evaluaron por día.

Las propiedades reológicas de la masa, se determinaron por medio de una prueba de tensión-relajación, utilizando un analizador de textura TA-XT2i (Stable Micro Systems, London, U.K.), con software Texture Expert Exceed, versión 2.64, equipado con una celda de carga de $25 \mathrm{~kg}$ y, como accesorio, un plato de compresión (P/100), de $100 \mathrm{~mm}$ de diámetro. Esta prueba, se aplicó a las masas del tratamiento control y con $1 \%$ de DATEM o SSL. La muestra, se moldeó en forma cilíndrica (29,3mm altura, 49,8mm de diámetro), se colocó sobre la base de aluminio directamente bajo el plato cilíndrico y se comprimió $6 \mathrm{~mm}$ de su altura original (nivel de deformación de $20 \%$ ), con una velocidad de ensayo de $10 \mathrm{~mm} / \mathrm{s}$. Una deformación constante de compresión fue aplicada a la muestra por 120s. La base y la sonda, se lubricaron con parafina líquida, para minimizar efectos de fricción (Rodríguez-Sandoval et al. 2008). Cada prueba, se realizó con nueve réplicas y cada ensayo completo, se repitió dos veces. Los datos de las curvas de tensión-relajación, se ajustaron al modelo de Peleg, utilizando la Ecuación 1 (Peleg \& Normand, 1983).

$$
\frac{\sigma_{0} t}{\sigma_{0}-\sigma(t)}=k_{1}+k_{2} t
$$

Donde $\sigma(\mathrm{t})$ es el esfuerzo decreciente a cualquier tiempo de la prueba $(\mathrm{Pa}), \sigma_{0}$ es el esfuerzo inicial $(\mathrm{Pa})$, t es el tiempo (s), $\mathrm{k}_{1}$ (s) y $\mathrm{k}_{2}$ son constantes y representan la velocidad de de- caimiento inicial y el valor hipotético de la fuerza normalizada asintótica, respectivamente (Steffe, 1996).

El diámetro del buñuelo, se determinó con un calibrador digital (Absolute 500-196, Mitutoyo Inc., Sao Paulo, Brasil), con una sensibilidad de $\pm 0,001$ y la pérdida de peso en una balanza de precisión (Modelo BL-6205, Shimadzu Corp., Japón). Igualmente, se calculó el volumen utilizando el método de desplazamiento de semillas de mijo (López et al. 2004). El contenido de grasa, se midió por duplicado, utilizando el método de extracción soxhlet, basado en la NTC 668 (ICONTEC, 1973). La evaluación de la humedad de la miga, se realizó utilizando el método 44-19 de la AACC (2000) y la actividad de agua $\left(\mathrm{a}_{\mathrm{w}}\right)$, se cuantificó con un higrómetro de punto de rocío, a $25^{\circ} \mathrm{C}$ (Aqualab serie 3TE, Decagon, Devices, Pullman, WA, USA) (Cortes et al. 2007). Se reportó el valor promedio de tres determinaciones.

La determinación del color, se realizó con un espectrocolorímetro de esfera (SP60, X-Rite, USA), iluminante D65 y observador de $10^{\circ}$, como referencia. A partir del espectro de reflexión, se obtuvieron las coordenadas de color CIE L*a*b*, donde $L^{*}$ es un indicador de la luminosidad, $a^{*}$ indica la cromaticidad en el eje verde (-) a rojo $(+), y$ b* la cromaticidad en el eje azul (-) a amarillo (+) (Morales \& Arribas-Lorenzo, 2008). Los valores triestímulos $X Y Z$, que se obtienen del cálculo del valor espectral, iluminante y observador estándar, se transforman en las coordenadas de color CIE L*a*b* aplicando funciones cúbicas (X-Rite, 2002).

Las propiedades de textura del buñuelo, se llevaron a cabo en el analizador de textura, descrito previamente, usando la misma celda de carga. Los parámetros establecidos fueron: velocidad pre-ensayo $1 \mathrm{~mm} / \mathrm{s}$, velocidad de ensayo $1 \mathrm{~mm} / \mathrm{s}$ y velocidad pos-ensayo $2 \mathrm{~mm} / \mathrm{s}$. Para las pruebas, se dividió el buñuelo en tres partes y se utilizó la parte central para el análisis de perfil de textura (TPA) y los extremos para la prueba de punción. En cada formulación, se tomaron siete mediciones repetidas sobre muestras separadas y se reportaron los valores medios. En el TPA, se empleó una sonda cilíndrica de $12,7 \mathrm{~mm}$ de diámetro $(\mathrm{P} / 0,5 \mathrm{R})$, comprimiendo $5 \mathrm{~mm}$ de su altura inicial, con una velocidad de ensayo de $1 \mathrm{~mm} / \mathrm{s}$ y manteniendo 5 s entre los ciclos. Los principales parámetros texturales obtenidos fueron: dureza, cohesividad y elasticidad (Rodríguez-Sandoval et al. 2008). La prueba de punción, se utilizó como método objetivo, para medir la dureza de la corteza del buñuelo. Esta consistió en determinar la fuerza máxima de ruptura por ensayos de penetración, empleando una sonda cilíndrica de $2 \mathrm{~mm}$ de diámetro (P/2), a una velocidad constante de $1 \mathrm{~mm} / \mathrm{s}$ y una distancia de penetración de $8 \mathrm{~mm}$ (Lorenzo et al. 2008).

El diseño experimental empleado en este estudio fue un factorial, completamente aleatorio, utilizando tres factores: DA- 
TEM, SSL y tiempo; a tres niveles para DATEM y SSL: $0 \%$, $0,5 \%$ y $1 \%$ y cuatro niveles para el tiempo: $1,24,48$ y $72 \mathrm{~h}$. Se realizaron en total trece experimentos, con cinco repeticiones del punto central. El análisis estadístico, se realizó con Design Expert Versión 7.0.0 (Stat-Ease Inc., Minneapolis, MN, EE.UU.).

\section{RESULTADOS Y DISCUSIÓN}

Las propiedades de empastamiento muestran la intensidad de los cambios que se producen durante la modificación del almidón. La viscosidad máxima refleja la degradación molecular, mientras que la gelatinización refleja la integridad del gránulo (Rodríguez-Sandoval et al. 2012). El tratamiento tér- mico seguido de una acción mecánica sobre los gránulos de almidón conduce a cambios estructurales y, consecuentemente, pérdida de la integridad del gránulo. La intensidad de esta ruptura depende del tipo de almidón, temperatura y cizalla mecánica utilizados durante la gelatinización (Dias et al. 2011). Por ejemplo, al mezclar goma guar con almidón nativo de yuca crea un efecto sinérgico, afectando la fase continua y aumentando la fuerza de cizalla ejercida sobre los gránulos de almidón, hinchados en la curva de empastamiento, si se compara con una suspensión compuesta solamente de almidón y agua (Chaisawang \& Suphantharika, 2006). La tabla 1 muestra los resultados obtenidos de las curvas de empastamiento del almidón agrio de yuca.

Tabla 1. Resultados de las curvas de empastamiento del almidón agrio de yuca.

\begin{tabular}{|l|r|}
\hline \multicolumn{2}{|c|}{ Propiedades de las curvas de empastamiento } \\
\hline T gel ( $\left.{ }^{\circ} \mathrm{C}\right)$ & $69,57 \pm 0,12$ \\
\hline $\mathrm{V}$ max (RVU*) & $45,97 \pm 1,11$ \\
\hline $\mathrm{V}$ final (RVU*) & $14,92 \pm 0,30$ \\
\hline Inest. gel (Breakdown) (RVU*) & $33,75 \pm 0,66$ \\
\hline Asentamiento (Setback) (RVU*) & $4,11 \pm 0,46$ \\
\hline
\end{tabular}

*RVU: Unidad del viscoamilógrafo rápido

La temperatura inicial de gelatinización fue de $69,57^{\circ} \mathrm{C}$; este resultado es mayor que el hallado por Franco et al. (2010). Un rango de temperatura inicial de gelatinización de 58,8 $62,2^{\circ} \mathrm{C}$ ha sido reportado por Mestres et al. (2000). La viscosidad máxima fue más baja que lo reportado en la literatura para almidones agrios de yuca (Mestres et al. 2000; Franco et al. 2010; Maieves et al. 2011); esto se puede deber a las características del almidón analizado, debido, posiblemente, a un tiempo de fermentación superior, indicando una mayor degradación del almidón (Pereira et al. 2004); sin embargo, las diferencias en la concentración del almidón, para realizar la solución utilizada en este método, pueden afectar las propiedades de empastamiento, lo cual, sería una explicación para la diferencia de los resultados encontrados por otros autores (Maieves et al. 2011).

Onitilo et al. (2007) encontraron, para almidones agrios de diferentes variedades de yuca, valores de viscosidad final, entre 140,33 a 167,75 RVU; Inestabilidad del gel (Breakdown), entre 201,3 a 228,96 RVU y asentamiento (Setback), entre 33,58 a 41,54 RVU, propiedades que son superiores a las encontradas para el almidón agrio de yuca, evaluado en este estudio. El asentamiento es la tendencia a la retrogradación y permite evaluar la diferencia de viscosidad del gel durante la fase de enfriamiento. La retrogradación del almidón, se produce como resultado de la recristalización de las molé- culas de amilosa y amilopectina, a través de la formación de enlaces de hidrógeno nuevos, que resultan en la formación de geles. La gelatinización, la tendencia a la retrogradación y otras propiedades reológicas de los geles de almidón son respuestas a la naturaleza de los gránulos, contenido de amilosa y amilopectina, arreglo espacial de estos dos polisacáridos en la estructura interna y al grado de compactación (Lindeboom et al. 2004).

En las pruebas de tensión-relajación, las constantes de relajación están relacionadas con las características viscoelásticas de la masa del buñuelo. El coeficiente de correlación del modelo de Peleg, para todas las muestras, estuvo próximo a 1. En la mayoría de los materiales viscoelásticos, después de la aplicación de un esfuerzo constante, se observa una disminución en los valores de fuerza, necesarios para el mantenimiento de la deformación (Sozer et al. 2008). Las constantes del modelo de Peleg, se presentan en la tabla 2. Los valores de $\mathrm{k}_{1}$ y $\mathrm{k}_{2}$, se encuentran entre 0,85 a 1,12 y 1,024 a 1,037, respectivamente. El valor de $\mathrm{k}_{1}$, se vio afectado significativamente por la adición de cada uno de los emulsificantes, obteniendo los menores valores en las formulaciones que contenían DATEM $1 \%$ o SSL $1 \%$, lo que significa que la utilización de estos emulsificantes da lugar a una masa más líquida, con menos habilidad para almacenar energía. 
Tabla 2. Parámetros de tensión-relajación del Modelo de Peleg para muestras de masas de buñuelo, con 1\% SSL o 1\% DATEM.

\begin{tabular}{|l|c|c|}
\hline \multicolumn{1}{|c|}{ Muestras } & $\mathbf{k}_{\mathbf{1}}(\mathbf{s})^{{ }^{*}}$ & $\mathbf{k}_{2}{ }^{{ }^{*}}$ \\
\hline Control & $1,12 \pm 0,11^{\mathrm{a}}$ & $1,037 \pm 0,006^{\mathrm{a}}$ \\
\hline DATEM $1 \%$ & $0,91 \pm 0,16^{\mathrm{b}}$ & $1,024 \pm 0,006^{\mathrm{b}}$ \\
\hline SSL $1 \%$ & $0,85 \pm 0,14^{\mathrm{b}}$ & $1,025 \pm 0,007^{\mathrm{b}}$ \\
\hline
\end{tabular}

"Medias de al menos 9 replicados $\pm \mathrm{DE}$

Muestras de la misma columna con diferentes letras difieren significativamente $(\mathrm{p}<0.05)$.

El recíproco de $\mathrm{k}_{1}$ representa la velocidad de relajación inicial y es una medida de la facilidad con que el material se deforma. Valores más altos de $\mathrm{k}_{1}$ representan un material más sólido y más duro que disipa menos energía, requiriendo más fuerza para ser comprimido (Rodríguez-Sandoval et al. 2009). La constante $k_{2}$. se vio influenciada, significativamente, por el tipo de emulsificante. Muestras de masas adicionadas con DATEM $1 \%$ y SSL $1 \%$ presentaron valores más bajos, lo que significa que estas muestras de masa necesitan menos fuerza normalizada para lograr la misma deformación, que las de la formulación control. Estos resultados son menores a los obtenidos por Rodríguez-Sandoval et al. (2009), quienes reportaron valores de $\mathrm{k}_{1}$, entre 4,9 a 5,8 y k2, entre 1,6 a 2,2, para masas de yuca reconstituidas. Singh et al. (2006) hallaron valores de $\mathrm{k}_{1}$, entre 1,29 a 1,30 y $\mathrm{k}_{2}$, entre 1,27 a 1,33 , para quesos cheddar y mozzarella.
Los resultados experimentales de las propiedades físicas (peso, diámetro, volumen, color) y el contenido de grasa de muestras de buñuelo evaluadas después de $1 \mathrm{~h}$ de elaboración, se muestran en la tabla 3. El peso, el diámetro y el volumen no se vieron afectados significativamente por los emulsificantes. El volumen de los buñuelos fue superior a lo hallado en otra investigación, donde se reportaron volúmenes entre 9,64-15,28 $\mathrm{cm}^{3}$, para esferas de gluten fritas (Chiang et al. 2006). Los emulsificantes evaluados tampoco afectaron significativamente el contenido de grasa. El valor del contenido de grasa de los buñuelos, se encontró entre 16,67 a $20,43 \%$, lo que coincide con los resultados obtenidos en la literatura, reportándose valores de grasa entre 15,22 a $24,57 \%$, para un pasabocas frito a base de pasta de mango (Villamizar-Vargas \& Giraldo, 2010).

Tabla 3. Propiedades físicas y contenido de grasa del buñuelo, evaluado después de 1h de elaboración.

\begin{tabular}{|c|c|c|c|c|c|c|c|c|c|}
\hline Corrida & $\begin{array}{c}\text { DATEM } \\
\mathbf{( \% )}\end{array}$ & $\begin{array}{c}\text { SSL } \\
\mathbf{( \% )}\end{array}$ & $\begin{array}{c}\text { Peso } \\
\mathbf{( g )}\end{array}$ & $\begin{array}{c}\text { Diámetro } \\
\mathbf{( m m )}\end{array}$ & $\begin{array}{c}\text { Volumen } \\
\left(\mathbf{c m}^{\mathbf{3}} \mathbf{)}\right.\end{array}$ & $\begin{array}{c}\text { Conte- } \\
\text { nido } \\
\text { Grasa } \\
\mathbf{( \% )}\end{array}$ & L* & a* & b* \\
\hline 1 & 0 & 0 & 26,24 & 45,97 & 57,58 & 16,67 & 55,14 & 17,30 & 34,69 \\
\hline 2 & 1 & 0 & 26,56 & 44,56 & 52,17 & 17,99 & 57,19 & 15,89 & 33,19 \\
\hline 3 & 0 & 1 & 26,21 & 42,46 & 50,08 & 18,36 & 57,20 & 16,85 & 33,77 \\
\hline 4 & 1 & 1 & 26,38 & 41,91 & 42,83 & 20,43 & 57,06 & 17,24 & 34,67 \\
\hline 5 & 0 & 0,5 & 26,13 & 43,55 & 49,25 & 17,95 & 56,28 & 17,79 & 34,95 \\
\hline 6 & 1 & 0,5 & 26,26 & 42,60 & 45,08 & 18,33 & 56,90 & 17,43 & 35,47 \\
\hline 7 & 0,5 & 0 & 26,39 & 44,79 & 53,83 & 18,57 & 55,22 & 16,18 & 32,99 \\
\hline 8 & 0,5 & 1 & 26,14 & 42,64 & 48,92 & 18,72 & 57,75 & 16,21 & 34,32 \\
\hline 9 & 0,5 & 0,5 & 26,25 & 43,31 & 45,50 & 17,99 & 55,91 & 16,48 & 33,20 \\
\hline 10 & 0,5 & 0,5 & 26,50 & 43,52 & 53,67 & 18,10 & 57,10 & 16,93 & 34,81 \\
\hline 11 & 0,5 & 0,5 & 26,89 & 46,47 & 60,50 & 17,97 & 52,92 & 17,88 & 34,59 \\
\hline 12 & 0,5 & 0,5 & 26,34 & 43,35 & 46,17 & 18,20 & 52,40 & 17,31 & 33,92 \\
\hline 13 & 0,5 & 0,5 & 26,25 & 43,51 & 45,75 & 18,23 & 55,95 & 16,79 & 33,33 \\
\hline
\end{tabular}


El color es uno de los factores importantes en la calidad de los productos fritos. Los parámetros $L^{*}, a^{*}$ y b* no fueron afectados significativamente por los emulsificantes. Los valores de $L^{*}$ estuvieron entre 55,14 - 57,75, desplazándose al blanco; a* entre 15,89 - 17,79, tendencia hacia el rojo y b* entre 32,99 - 35,47, acercándose al amarillo, lo cual, da como resultado un color café, característico de este tipo de productos. Estos resultados difieren a lo reportado en la literatura para otros productos fritos, debido a la diferencia en la formulación (harinas de trigo, soya, arroz, entre otros) y condiciones de proceso (Chiang et al. 2006; Allais et al. 2006; Alpaslan \& Hayta, 2010).

En cuanto a la actividad de agua y contenido de humedad de la miga de las muestras evaluadas durante el almacenamiento, se observó que la influencia del tiempo es altamente significativa $(p<0,05)$, mientras que los emulsificantes no afectaron significativamente estas variables. El contenido de humedad y de actividad de agua de la miga, disminuyeron a lo largo del tiempo de almacenamiento, como se muestra en la figura 1. Esto está de acuerdo con los resultados obtenidos por Xu et al. (1992) y Sidhu et al. (1997), en panes. Esta disminución de la humedad parece ser causada, principalmente, por la redistribución del agua, es decir, la migración de la humedad de la miga a la corteza. Por su parte, la disminución de la actividad de agua podría ser causada por el agua libre enlazada con sustancias hidrofílicas, como el almidón (Wang et al. 2011).

Los resultados del ensayo de punción no fueron influenciados significativamente por los emulsificantes y variaron solamente con el tiempo de almacenamiento, mostrando una


Figura 1. Variación del contenido de humedad y de actividad de agua $\left(\mathrm{a}_{\mathrm{w}}\right)$, de los buñuelos durante el almacenamiento.

significativa disminución $(\mathrm{p}<0,05)$ de la fuerza necesaria para romper el producto, sobre todo en las primeras $24 \mathrm{~h}$; luego, permanece aproximadamente constante (Figura 2) y esto se debe a la migración de la humedad de la miga hacia la corteza, haciéndola más blanda.

En cuanto a las propiedades obtenidas del análisis de perfil de textura, la dureza de la miga aumentó significativamente $(p<0,05)$ durante el almacenamiento (Figura 2). El endurecimiento de la miga es un fenómeno complejo causado por fenómenos simultáneos. Entre ellos, la retrogradación del almidón, siendo el más importante; sin embargo, la recristalización de amilosa, la perdida y redistribución del contenido humedad, también contribuyen al incremento de la dureza de la miga del buñuelo (Bárcenas \& Rosell, 2007). No se observa un efecto claro de los emulsificantes sobre la dureza del producto.
La elasticidad y la cohesividad de las muestras mostraron comportamientos similares y una dependencia significativa $(p<0,05)$ entre los dos emulsificantes, para estas variables. Con el transcurso del tiempo de almacenamiento, los buñuelos se volvieron menos cohesivos y elásticos (Figura 3) y se registró, además, una dependencia significativa $(\mathrm{p}<0,05)$ de ambas variables, con el contenido de SSL. Al aumentar la concentración de dicho emulsificante, la cohesividad y la elasticidad disminuyeron significativamente $(p<0,05)$ (Figura 3). El comportamiento de las muestras con DATEM fue muy parecido a las adicionadas con SSL, razón por la cual, solamente se muestran los resultados con este último emulsificante.

El almidón agrio de yuca utilizado posee alta temperatura de empastamiento y viscosidad máxima y baja inestabilidad del gel y asentamiento. Los valores de $\mathrm{k}_{1}$ y $\mathrm{k}_{2}$ reportados en un 


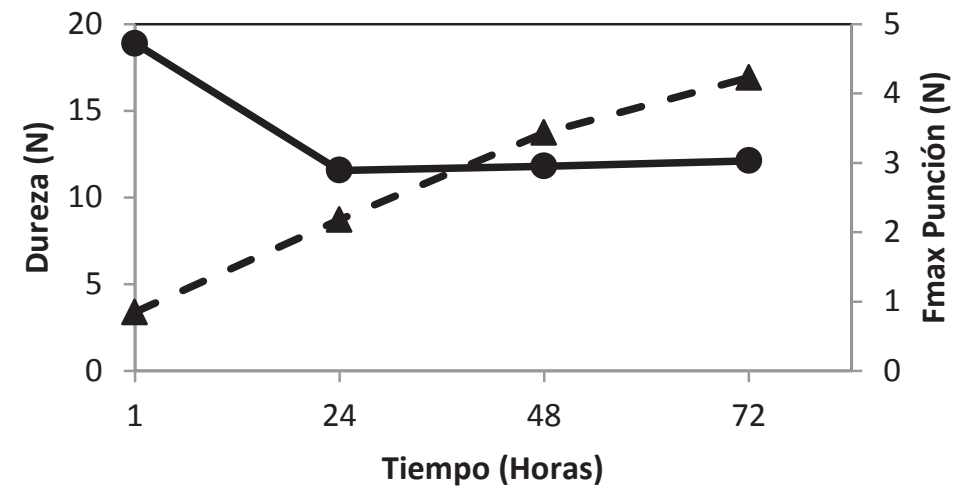

- Dureza (N) Fmax Punción (N)

Figura 2. Variación de la fuerza máxima de punción y de dureza de los buñuelos, durante el almacenamiento.
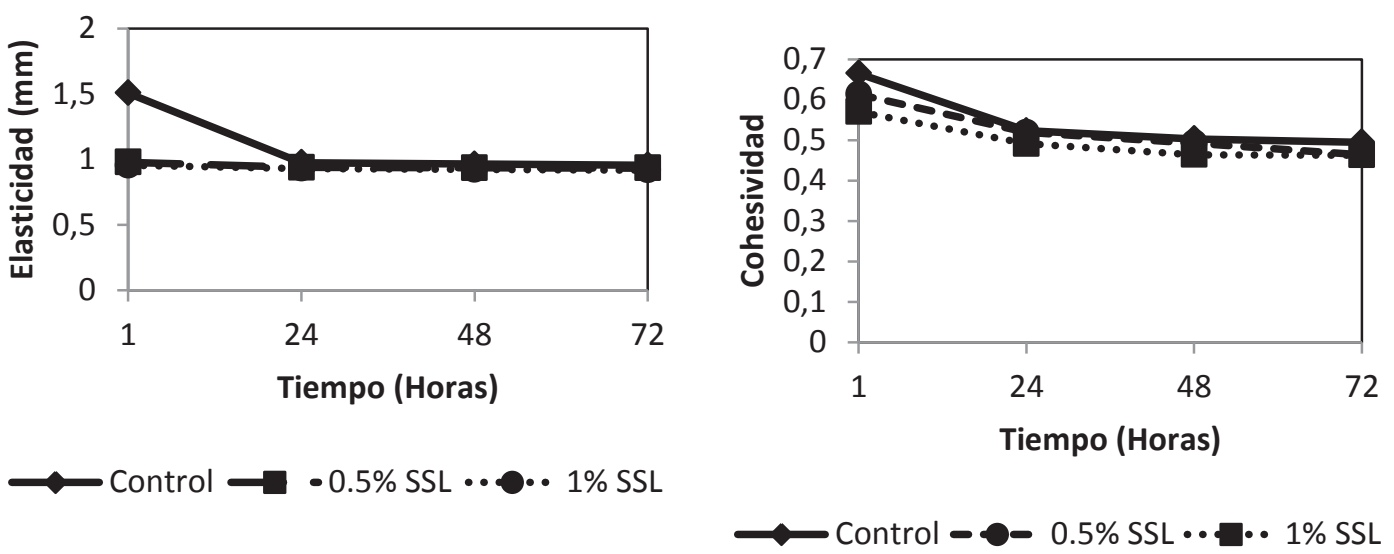

Figura 3. Efecto del emulsificante SSL sobre la elasticidad y la cohesividad de los buñuelos, durante el almacenamiento.

intervalo de 0,85 a 1,12 y 1,024 a 1,037 , respectivamente, corroboran la naturaleza viscoelástica de la masa de buñuelo elaborado con DATEM o SSL. La calidad del buñuelo no fue mejorada con la adición de los emulsificantes DATEM y SSL. No se observó influencia significativa de los emulsificantes en las características físicas, fisicoquímicas y de textura, como la punción y la dureza. Únicamente se presentó una interacción significativa entre los dos emulsificantes, para las variables de cohesividad y de elasticidad. El factor tiempo afectó significativamente el contenido de humedad, actividad de agua, de punción, de dureza, de cohesividad y de elasticidad. Investigaciones futuras podrían incluir el uso individual o combinado de otros aditivos, como hidrocoloides que, posiblemente, mejorarán las propiedades de calidad del buñuelo, durante el almacenamiento. Además, se recomienda aplicar pruebas de reología dinámica, para evaluar el efecto de los aditivos sobre las características de la masa y estudiar la estructura del producto, con técnicas de microscopia electrónica.

Agradecimientos: Los autores agradecen a la empresa Nutring S.A. (Buenos Aires, Argentina), por la donación de los emulsificantes utilizados en este estudio. Conflicto de intereses: El manuscrito fue preparado y revisado con la participación de todos los autores, quienes declaramos que no existe ningún conflicto de interés, que ponga en riesgo la validez de los resultados.

\section{BIBLIOGRAFÍA}

1. ALLAIS, I.; EDOURA-GAENA, R.B.; DUFOUR, E. 2006. Characterisation of lady finger batters and biscuits 
by fluorescence spectroscopy-relation with density, color and texture. J. Food Eng. 77(4):896-909.

2. ALPASLAN, M.; HAYTA, M. 2010. Effect of soy flour, rice flour and semolina supplementation on the textural and sensory properties of dough and a deep-fried product. J. Food Proc. Pres. 34:490-500.

3. AMERICAN ASSOCIATION OF CEREAL CHEMISTS AACC-. 2000. Approved Methods of Analysis, 10th ed. Method 44-19. Moisture -- Air-Oven Method, Drying at $135^{\circ}$. AACC International, St Paul, MN, USA.

4. ARISTIZÁBAL, J.; SÁNCHEZ, T. 2007. Guía técnica para producción y análisis de almidón de yuca. Boletín de Servicios Agrícolas de la FAO. No. 163. Roma, Italia: Organización de Las Naciones Unidas para la Agricultura y la Alimentación (FAO). 153p.

5. BÁRCENAS, M.E.; ROSELL, C.M. 2007. Different approaches for increasing the shelf life of partially baked bread: Low temperatures and hydrocolloid addition. Food Chem. 100:1594-1601.

6. BELTRÁN-OROZCO, M.C.; RENDÓN-MEZA, J.H.; GALLARDO-VELÁZQUEZ, T. 2007. Cinética de las características físicas de mantecadas bajas en grasa almacenada en dos tipos de material de empaque durante su vida de anaquel. Inf. Tecnol. 18(3):13-22.

7. CHAISAWANG, M.; SUPHANTHARIKA, M. 2006. Pasting and rheological properties of native and anionic tapioca starches as modified by guar gum and xanthan gum. Food Hydrocolloid. 20:641-649.

8. CHIANG, S.H.; CHEN, C.S.; CHANG, C.Y. 2006. Effect of wheat flour protein compositions on the quality of deep-fried gluten balls. Food Chem. 97(4):666-673.

9. CHIN, N.L.; GOH, S.K.; RAHMAN, R.A.; HASHIM, D.M. 2007. Functional effect of fully hydrogenated palm oil-based emulsifiers on baking performance of white bread. Int. J. Food Eng. 3(3):1-15.

10. CORTES, M.; GARCÍA, A.; SUÁREZ, H. 2007. Fortificación de hongos comestibles (Pleurotus ostreatus) con calcio, selenio y vitamina C. Vitae. 14(1):16-24.

11. DIAS, A.R.G.; ZAVAREZE, E.R.; ELIAS, M.C.; HELBIG, E.; DA SILVA, D.O.; CIACCO, C.F. 2011. Pasting, expansion and textural properties of fermented cassava starch oxidised with sodium hypochlorite. Carbohydr. Polym. 84(1):268-275.
12. FRANCO, C.M.L.; OGAWA, C.; RABACHINI, T.; ROCHA, T.; CEREDA, M.P.; JANE, J. 2010. Effect of lactic acid and UV irradiation on the cassava and corn starches. Braz. Arch. Biol. Technol. 53(2):443-454.

13. GOMES-RUFFI, C.R.; CUNHA, R.H.; ALMEIDA, E.L.; CHANG, Y.K.; STEEL, C.J. 2012. Effect of the emulsifier sodium stearoyl lactylate and of the enzyme maltogenic amylase on the quality of pan bread during storage. LWT-Food Sci. Technol. 49(1):96-101.

14. INSTITUTO COLOMBIANO DE NORMAS TÉCNICAS Y CERTIFICACIÓN -ICONTEC-. 1973. Alimentos y Materias Primas. Determinación de los contenidos de grasa y fibra cruda. NTC 668. Bogotá D.C.: ICONTEC. 8p.

15. KOOCHEKI, A.; MORTAZAVI, S.A.; MAHALATI, M.N.; KARIMI, M. 2009. Effect of emulsifiers and fungal a-amylase on rheological characteristics of wheat dough and quality of flat bread. J. Food Proc. Eng. 32:187-205.

16. LINDEBOOM, N.; CHANG, P.R.; TYLER, R.T. 2004. Analytical, biochemical and physicochemical aspects of starch granule size, with emphasis on small granule starches: a review. Starch/Stärke. 56(3-4):89-99.

17. LÓPEZ, A.C.B.; PEREIRA, A.J.G; JUNQUEIRA, R.G. 2004. Flour mixture of rice flour, corn and cassava starch in the production of gluten-free white bread. Braz. Arch. Biol. Technol. 47(1):63-70.

18. LORENZO, G.; ZARITZKY, N; CALIFANO, A. 2008. Optimization of non-fermented gluten-free dough composition based on rheological behavior for industrial production of "empanadas" and pie-crusts. J. Cereal Sci. 48:224231.

19. MESTRES, C.; BOUNGOU, O.; AKISSOE, N.; ZAKHIA, N. 2000. Comparison of the expansion ability of fermented maize flour and cassava starch during baking. J. Sci. Food Agric. 80:665-672.

20. MILDE, L.B.; GONZÁLEZ, K.G.; VALLE, C.; RYBAK, A. 2009. Pan de fécula de mandioca con leche. Comportamiento físico al adicionar un emulsionante. Rev. Cienc. Tecnol. 11:4-8.

21. MORALES, F.J.; ARRIBAS-LORENZO, G. 2008. The formation of potentially harmful compounds in churros, a Spanish fried-dough pastry, as influenced by deep frying conditions. Food Chem. 109:421-425. 
22. MAIEVES, H.A.; OLIVEIRA, D.C.D.; FRESCURA, J.R.; AMANTE, E.R. 2011. Selection of cultivars for minimization of waste and of water consumption in cassava starch production. Ind. Crop Prod. 33(1):224228.

23. ONITILO, M.O.; SANNI, L.O.; OYEWOLE, O.B.; MAZIYADIXON, B. 2007. Physicochemical and functional properties of sour starches from different cassava varieties. Int. J. Food Prop. 10(3):607-620.

24. ONYANGO, C.; UNBEHEND, G; LINDHAUER, M. G. 2009. Effect of cellulose-derivatives and emulsifiers on creep-recovery and crumb properties of glutenfree bread prepared from sorghum and gelatinized cassava starch. Food Res. Int. 42(8):949-955.

25. PELEG, M.; NORMAND, M.D. 1983. Comparison of two methods for stress relaxation data presentation of solid foods. Rheol. Acta. 22:108-113.

26. PEREIRA, J.; CIACCO, C.F.; VILELA, E.R.; PEREIRA, R. 2004. Função dos ingredientes na consistência da massa e nas características do pão de queijo. Ciênc. Tecnol. Aliment. 24(4):494-500.

27. RODRÍGUEZ-SANDOVAL, E.; FERNÁNDEZ-QUINTERO, A.; ALONSO-ALCALÁ, L.; OSPINA-PATIÑO, B. 2006. Reología de suspensiones preparadas con harina precocida de yuca. Ing. Desarr. 19:17-30.

28. RODRÍGUIEZ-SANDOVAL, E.; FERNÁNDEZ, A.; SANDOVAL, A; QUICAZÁN, M.C. 2008. Effect of cooking time and storage temperature on the textural properties of cassava dough. J. Texture Stud. 39:68-82.

29. RODRÍGUEZ-SANDOVAL, E.; FERNÁNDEZ-QUINTERO, A.; CUVELIER, G. 2009. Stress relaxation of reconstituted cassava dough. LWT-Food Sci. Technol. 42:202-206.

30. RODRÍGUEZ-SANDOVAL, E.; SANDOVAL, G.; CORTESRODRÍGUEZ, M. 2012. Effect of quinoa and potato flours on the thermomechanical and breadmaking properties of wheat flour. Braz. J. Chem. Eng. 29(03):503-510.
31. SCIARINI, L.S.; RIBOTTA, P.D.; LEÓN, A.E.; PÉREZ, G.T. 2012. Incorporation of several additives into gluten free breads: Effect on dough properties and bread quality. J. Food Eng. 111(4):590-597.

32. SIDHU, J.S.; AL-SAQER, J.; AL-ZENKI, S. 1997. Comparison of methods for the assessment of the extent of staling in bread. Food Chem. 58:161-167.

33. SINGH, H.; ROCKALL, A.; MARTIN, C.R.; CHUNG, O.K.; LOOKHART, G.L. 2006. The analysis of stress relaxation data of some viscoelastic foods using a texture analyzer. J. Texture Stud. 37(4):383-392.

34. SOZER, N.; KAYA, A.; COSKUN DALGIC, A. 2008. The effect of resistant starch addition on viscoelastic properties of cooked spaghetti. J. Texture Stud. 39:1-16.

35. STAMPFLI, L.; NERSTEN, B. 1995. Emulsifiers in bread making. Food Chem. 52(4):353-360.

36. STEFFE, J.F. 1996. Rheological methods in food process engineering, (2nd ed.). East Lansing, MI, USA: Freeman Press. 418p.

37. VILLAMIZAR-VARGAS, R.H.; GIRALDO, G.A. 2010. Obtención y caracterización de un pasabocas a partir de una pasta a base de mango mediante fritura por inmersión. Rev. Tumbaga. 1(5):149-164.

38. XU, A.; CHUNG, O.K.; PONTE, J.G. 1992. Bread crumb amylograph studies. I. Effects of storage time, shortening, flour lipids, and surfactants. Cereal Chem. 69:495-501.

39. X-RITE. 2002. Guía para entender la comunicación del color. Grandville, MI: X-Rite Incorporated. 26 p.

40. WANG, T.C.; LIN, SC.; SHEN, Y.P.; WANG-MCCALL, T.L.; CHIN, M.H.; LAN, K.P.; CHENG, P.Y.; YANG, C.C. 2011. Studies of retarding agent for decreasing starch retrogradation in wankao (rice curd). J. Food Quality. 34:268-277.

Recibido: Enero 31 de 2013

Aceptado: Octubre 15 de 2013

Como citar:

Morales, M.M.; Rodríguez-Sandoval, E.; Cortes, M. 2013. Efecto de los emulsificantes sobre las características físicas y texturales del buñuelo. Rev. U.D.C.A Act. \& Div. Cient. 16(2): 417-425. 